\title{
A Focus on High-Temperature Shape Memory Alloys
}

\author{
Hans J. Maier ${ }^{1}$ iD
}

Published online: 7 December 2015

(C) ASM International 2015

There is growing interest in high-temperature shape memory alloys both from academia and industry, as this class of smart materials can deliver large reversible strains at elevated temperatures. Target applications are in aerospace, automotive, energy exploration, and conversion industries, where the operating temperatures are often well above $100{ }^{\circ} \mathrm{C}$. Some of the early high-temperature shape memory alloys already showed excellent properties, but relied mostly on substantial amounts of precious constituents. Clearly, such materials are not suited for the mass market. Lately, however, new alloys have been developed that are promising both in terms of performance and cost. Still, it is fair to say that this research area is in its infancy as compared to conventional shape memory alloys. Currently a major roadblock is microstructural instability at elevated temperatures, and advanced experimental techniques are needed to shed light on the underlying mechanisms, which eventually cause functional degradation. Similarly, theoretical efforts aim at developing validated models that allow for predicting the behavior of these materials under actual service conditions. The current special issue focuses on recent advances and challenges in this rapidly evolving research field. Consequently, the peer-reviewed papers address a wide range of topics from alloy development and processing to characterization and

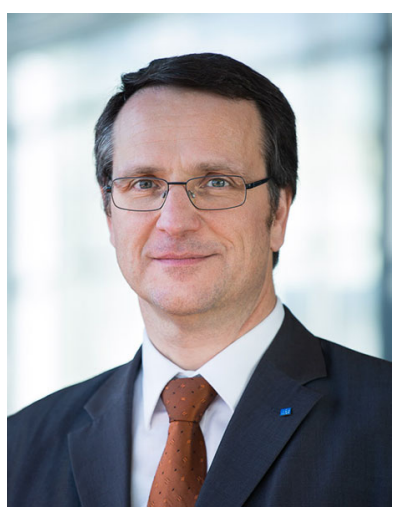

testing, and last but not least modeling of high temperature shape memory alloys. A single issue can by no means provide for a complete coverage of the field. What makes this issue special is that the papers provide for both an excellent overview of the current state-of-the-art and highlight directions for future research. Sincere thanks goes to the many people that have put a lot of time and effort into this special issue. The team of Shape Memory and Superelasticity wishes you an enjoyable read.

Hans J. Maier

maier@iw.uni-hannover.de

1 Institut für Werkstoffkunde (Materials Science), Leibniz Universität Hannover, Hannover, Germany 